the foot. The Spearman correlation coefficient between the DECT urate volume and the number of joints with a positive DC sign was 0.15 .

Conclusions: US and DECT do not provide the same assessment of tophus size. The correlation is improved when considering tophi localized in the feet. The number of joints with positive DC sign does not correlate to the volume of urate deposition in the soft tissues measured by DECT.

Disclosure of Interest: None declared

DOI: 10.1136/annrheumdis-2017-eular.4118

\section{THU0403 URATE-LOWERING TREATMENT AND RISK OF INCIDENT UROLITHIASIS IN PEOPLE WITH GOUT: A NESTED CASE-CONTROL STUDY}

C.-F. Kuo, J.-S. Chen. Division of Rheumatology, Allergy and Immunology, Chang Gung Memorial Hospital, Taoyuan, Taiwan, Province of China

Background: A higher risk of urolithiasis has been reported in gout patients. However, whether urate-lowering treatments, including both xanthine oxidase inhibotors and uricosuric agents, are beneficial to reduce the risk or urolithiasis in gout patients has not been examined.

Objectives: To investigate the independent associations between urate-lowering treatment (ULT) and the risk of urolithiasis in incident gout patients.

Methods: We conducted a nested case-control study based on the Taiwan National Health Insurance Research Database (NHIRD), which was used to identify 473,858 newly diagnosis gout patients during the period from January 1 , 2000 through December 31, 2004. All these patients were followed until December 31,2013 . We considered patients who first diagnose incident urolithiasis after the date of entry cohort (gout onset) as cases and the diagnostic date was defined as index date. Each case was matched up to five eligible controls whose follow-up period included the case's index date by sex, birth of year and gout diagnosis year. And the index date of case was assigned to the matched controls. Odds ratios (ORs) and $95 \%$ confidence interval $(\mathrm{Cl})$ of urolithiasis associated with cumulative defined daily dose (cDDD) of xanthine oxidase inhibitor and uricosuric agents were main ourcome measures.

Results: Gout patients with incident urolithiasis $(n=32,654)$ occurring after the initial diagnosis of gout aged 20-79 were age- and sex-matched 1:5 to 163,270 gout patients without urolithiasis. After adjusting for age, sex, urbanization status, income, occupation, and pertinent drugs and comorbidities, the OR of urolithiasis associated with use of ULT among gout patients were $1.04(95 \% \mathrm{Cl} 1.00$ to 1.07$)$ for those with 28-90 cDDD, 0.95 (95\% confidence interval 0.91-0.99) for 91-365 cDDD and 0.77 (95\% confidence interval $0.73-0.82)$ for $>365$ cDDD, compared with those with a cDDD $<28$. The OR $(95 \% \mathrm{Cl})$ for urolithiasis associated with xanthine oxidase inhibitor use was $0.94(0.89-0.99)$ for 28-90 cDDD, 0.90 $(0.84-0.97)$ for $91-365 \mathrm{cDDD}$ and $0.73(0.63-0.83)$ for $>365 \mathrm{cDDD}$. For uriscouric agents, the OR $(95 \% \mathrm{Cl})$ for urolithiasis among those with 28-91, 91-365 and $<365$ cDDD were $1.04(1.01,1.08), 0.94(0.90-0.98)$ and $0.78(0.73-0.84)$, respectively.

Conclusions: Higher ULT consumption was associated with a lower risk of urolithiasis. Xanthine oxidase inhibitors associated with reduced urolithiasis risk consistently across the range of consumption studied but for uricosuric agents inadequate cumulative dose results in a higher risk despite the risk reduced gradually with a higher cumulative dose.

Disclosure of Interest: None declared

DOI: 10.1136/annrheumdis-2017-eular.2913

\section{THU0404 SELF-REPORTED SEVERITY OF GOUT IN A PRIMARY CARE SETTING AND ASSOCIATED FACTORS - RESULTS FROM A GOUT SURVEY IN PRIMARY CARE}

A.J. Landgren, M. Dehlin, U. Bergsten, V. Sigurdardottir, P. Drivelegka, T. Zverkova Sandström, L. Jacobsson. Department of Rheumatology and Inflammation Research, Institution of Medicine, Sahlgrenska Academy. University of Gothenburg., Gothenburg, Sweden

Background: Patients with gout are usually seen in primary care. There are numerous reports regarding severity of gout in cohort studies and patients seen in more specialized care, less is known about the spectrum of severity of the disease in primary care.

Objectives: To describe the pattern of self-reported disease severity of gout and predictors thereof in a primary care setting.

Methods: All patients above 18 with an ICD10-diagnosis of gout at a health care visit in primary care (Jan 2015 through Aug 2016) were identified from primary care within the Western Sweden Health Care Region (WSHCR). 941 patients were identified and included in the study. They were sent a questionnaire with questions regarding comorbidities, demographics, gout characteristics and Health Assessment questionnaire ( $\mathrm{HAQ})$ reflecting disability. Self-reported disease severity was assessed by a three-level Likert scale (mild, moderate, severe). Possibly associated factors were analyzed by bivariate logistic regression (binary outcome: mild vs. moderate/severe) models and factors that were statistically significant were further analyzed by multivariate analysis.

Results: The response rate was $54 \%$. Response rates were significantly lower in women overall and in men under the age of 50 . Covariates that were significantly associated with more severe self-reported severity of gout in the bivariate logistic regression models (Table 1) were: female sex, hyperlipidemia, higher number of previous attacks, attack during last month, HAQ. In the multivariate analysis objective measures of gout severity such as more than ten previous attacks and attack during last month were strongly associated with the patients grading of severity, in addition to presence of hyperlipidemia (Table 1).

Table 1. Characteristics of gout patients stratified by disease severity and logistic regression models

\begin{tabular}{|c|c|c|c|c|c|}
\hline Covariates & $\begin{array}{l}\text { Mild }^{*} \\
\mathrm{n}=272\end{array}$ & $\begin{array}{c}\text { Moderate }^{*} \\
n=130\end{array}$ & $\begin{array}{c}\text { Severe }^{*} \\
n=62\end{array}$ & $\begin{array}{c}\text { Univariate analyses ** } \\
\text { OR }(95 \% \mathrm{Cl}) \#\end{array}$ & $\begin{array}{c}\text { Multivariate analyses } \\
\text { (OR }(95 \% \mathrm{Cl}) \#\end{array}$ \\
\hline Age in years (mean) & 70.8 & 71.9 & 71.8 & $1.01(0.99-1.02)$ & $\mathrm{N} / \mathrm{A}$ \\
\hline Female sex $(\%)$ & 12.9 & 18.5 & 22.6 & $1.67(1.01-2.76)$ & $1.54(0.85-2.80)$ \\
\hline Education $\leq 9$ years $(\%)$ & 24.8 & 27.6 & 46.6 & $1.46(0.97-2.21)$ & $\mathrm{N} / \mathrm{A}$ \\
\hline Diabetes (\%) & 22.8 & 27.1 & 27.4 & $1.29(0.84-1.98)$ & N/A \\
\hline Kidney disease (\%) & 6.6 & 9.3 & 11.3 & $1.59(0.81-3.12)$ & N/A \\
\hline BMI (mean) & 27.9 & 28.2 & 27.8 & $1.02(0.97-1.06)$ & N/A \\
\hline Hyperlipidemia (\%) & 29.4 & 44.2 & 46.8 & $2.00(1.36-2.95)$ & $2.13(1.39-3.28)$ \\
\hline \multicolumn{6}{|c|}{ Duration of gout in years } \\
\hline \multicolumn{6}{|c|}{ Total number of attacks (\%): } \\
\hline 1 & 19.5 & 6.4 & 12.1 & ref & ref \\
\hline $2-5$ & 50.0 & 40.8 & 39.7 & $1.93(1.01-3.67)$ & $1.51(0.77-2.96)$ \\
\hline $6-10$ & 14.8 & 20.8 & 13.8 & $2.98(1.42-6.25)$ & $2.12(0.97-4.62)$ \\
\hline$>10$ & 15.6 & 32.0 & 34.5 & $5.00(2.48-10.09)$ & $3.15(1.49-6.65)$ \\
\hline $\begin{array}{l}\text { Attack during the last } \\
\text { month }(\%)\end{array}$ & 11.5 & 30.4 & 30.6 & $3.43(2.10-5.61)$ & $1.81(1.36-2.41)$ \\
\hline $\operatorname{ULT}^{\mathbb{f}}(\%)$ & 46.3 & 50.8 & 50.0 & $0.85(0.46-1.55)$ & N/A \\
\hline $\mathrm{HAQ}^{\S}$ (mean) & 0.24 & 0.29 & 0.51 & $1.56(1.09-2.24)$ & $1.20(0.80-1.81)$ \\
\hline Tophus (\%) & 14.3 & 21.3 & 4.8 & $1.17(0.69-1.98)$ & $N / A$ \\
\hline
\end{tabular}

${ }^{*}$ Self-reported disease severity. ${ }^{* \star}$ ORs reflects yes vs no for dichotomous covariates, per year for age and duration and per unit for HAQ. \#Moderate/severe vs mild, "Urate-lowering therapy. Health assessment questionnaire.

Conclusions: Over $40 \%$ of patients with gout in a primary care setting rated their disease as moderate or severe. The validity of the patients rating was supported by other covariates reflecting disease severity. The observations that women and those with hyperlipidemia reported a more severe disease needs to be further explored.

Disclosure of Interest: None declared

DOI: 10.1136/annrheumdis-2017-eular.3452

\section{THU0405 KILLING TWO BIRDS WITH ONE STONE? THE DASH DIET AND THE RISK OF GOUT: 26-YEAR FOLLOW-UP OF A PROSPECTIVE COHORT}

S.K. Rai ${ }^{1}$, T.T. Fung ${ }^{2,3}$, N. Lu ${ }^{1}$, S.F. Keller ${ }^{1}$, G.C. Curhan ${ }^{4,5}$, H.K. Choi ${ }^{1,4}$ ${ }^{1}$ Division of Rheumatology, Allergy, and Immunology, Massachusetts General Hospital; ${ }^{2}$ Department of Nutrition, Harvard T.H. Chan School of Public Health; ${ }^{3}$ Department of Nutrition, Simmons College; ${ }^{4}$ Channing Division of Network Medicine, Department of Medicine, ${ }^{5}$ Renal Division, Department of Medicine, Brigham and Women's Hospital, Boston, United States

Background: The Dietary Approaches to Stop Hypertension (DASH) diet, which reduces blood pressure and is recommended in cardiovascular disease (CVD) $(1,2)$, has also been found to lower serum uric acid (SUA) levels, particularly among those with hyperuricemia (3). Thus, the DASH diet may be particularly useful in gout care by reducing both SUA and CVD risk, especially among patients with hypertension which affects $74 \%$ of gout patients (4), thereby "killing two birds with one stone". However, corresponding data for the risk of gout are not available. In contrast, a Western dietary pattern may increase the risk of gout.

Objectives: To prospectively examine the relation between the DASH and Western dietary patterns and the risk of gout among men.

Methods: Using the Health Professionals Follow-up Study, we prospectively examined the relation between the DASH and Western diets and incident gout in 44,444 male participants with no history of gout at baseline. Using validated food frequency questionnaires, each participant was assigned a DASH score (reflecting high intake of fruits, vegetables, nuts and legumes, low-fat dairy products, and whole grains, and low intake of sodium, sweetened beverages, and red and processed meats) and a Western pattern score (reflecting higher intake of red and processed meats, French fries, refined grains, sweets, and desserts). We identified incident cases of gout meeting the preliminary ACR survey criteria for gout, adjusting for potential confounders including age, total energy intake, body mass index (BMI), diuretic use, history of hypertension, history of renal failure, and intake of alcohol and coffee. We conducted stratified analyses to evaluate whether the association between the DASH and Western pattern scores and the risk of gout varied according to $\mathrm{BMI}$, alcohol use, and hypertension status.

Results: During 26 years of follow-up, we documented 1,731 confirmed cases of incident gout. A higher DASH score was associated with a lower risk for gout (multivariable relative risk [RR] for extreme quintiles, 0.68 [95\% confidence interval, 0.57 to 0.80 ]; $P$ for trend $<0.001$ ) (Table 1 ). In contrast, a higher Western pattern score was associated with an increased risk for gout (multivariable RR for extreme quintiles, 1.42 [95\% confidence interval, 1.16 to 1.74 ]; $P$ for trend $=0.005$ ) (Table 1). These associations persisted regardless of $\mathrm{BMI}$, alcohol use, and hypertension status, and there was no significant interaction with these variables (all $\mathrm{P}$ for interaction $>0.17$ ) 